\title{
Mapeamento da safena interna com ecocolor Doppler no pré-operatório de cirurgia de revascularização miocárdica
}

\author{
Fanilda Souto BARROS*, Sandra Maria PONTES*, Melchior Luiz LIMA**, José Silva HENRIQUE**, \\ Márcio Luiz ROLDI**, Fábio REIS**, José CARONE Jr. ${ }^{* *}$, Schariff MOISÉS**
}

RBCCV 44205-472

\begin{abstract}
Barros F S, Pontes S M, Lima M L, Henrique J S, Roldi M L, Reis F, Carone Jr. J, Moisés S - Mapeamento da safena interna com ecocolor Doppler no pré-operatório de cirurgia de revascularização miocárdica. Rev Bras Cir Cardiovasc 1999; 14 (4): 303-7.
\end{abstract}

RESUMO: Introdução: O advento do ecocolor Doppler vascular tornou possível informar o estado anatômico e funcional da safena interna ao cirurgião cardiovascular, no pré-operatório da cirurgia de revascularização miocárdica, sem riscos para o paciente ou para o conduto venoso. O objetivo do estudo foi, juntamente com o cirurgião, avaliar a utilidade do mapeamento da safena interna no pré-operatório.

Casuística e Métodos: Foram estudados, entre março de 1995 e maio de 1998, 208 extremidades inferiores de 104 pacientes selecionados para cirurgia de revascularização miocárdica. O critério utilizado para a seleção da safena foi a safena patente e o diâmetro maior ou igual a 3,0 mm, utilizando os aparelhos de ultra-sonografia ATL - HDI 3000 e Acuson-Sequóia 512.

Resultados: Dentre as 208 extremidades estudadas, $186(89,4 \%)$ das safenas preenchiam os critérios e a análise do cirurgião estava de acordo com a descrição do ecocolor Doppler. Dezessete $(8,2 \%)$ não preenchiam os critérios e, em 5 (2,4\%) ,o exame foi compatível com safenectomia radical. Das 17 safenas consideradas não apropriadas, 2 foram exploradas cirurgicamente, sendo a análise do cirurgião concordante com o ultra-som.

Conclusão: Os autores concluem que o ecocolor Doppler, sendo um método diagnóstico não invasivo, pode ser útil no mapeamento da safena interna no pré-operatório de revascularização miocárdica devido à sua capacidade de identificar e selecionar com segurança o vaso a ser utilizado como conduto vascular, o que permite ao cirurgião cardíaco o planejamento cirúrgico.

DESCRITORES: Revascularização miocárdica. Ecocardiografia Doppler em cores. Veia safena, ultrasonografia. Cuidados pré-operatórios, métodos.

\author{
INTRODUÇÃO \\ É antiga a preocupação do cirurgião cardiovas- \\ cular a respeito da qualidade do conduto vascular \\ a ser utilizado nas operações de revascularização. \\ Durante muitos anos, o método disponível para a \\ seleção de veias era a venografia. Esse método,
}

além da desvantagem de ser invasivo, apresentava o risco de provocar trombose da safena (1). A partir da década de 80, a ultra-sonografia tornou-se uma alternativa à venografia, identificando o vaso ${ }^{(2)}$. Com o avanço tecnológico, o mapeamento venoso, por meio do ecocolor Doppler, vem se mostrando de grande utilidade no planejamento cirúrgico dos pa-

\footnotetext{
Trabalho realizado no Instituto de Cardiologia do Espírito Santo e Angiolab - Laboratório de Diagnóstico Vascular de Vitória, ES, Brasil.

Apresentado no $26^{\circ}$ Congresso Nacional de Cirurgia Cardíaca. Fortaleza, CE, 8 a 10 de abril, 1999.

* Do Angiolab - Laboratório de Diagnóstico Vascular de Vitória - E.S e ICES

** Do Instituto de Cardiologia do Espírito Santo (ICES)

Endereço para correspondência: Fanilda Souto Barros. Angiolab - Diagnóstico Vascular. Av. Carlos G. de Sá, 60. Mata da Praia, Vitória, ES, Brasil. CEP: 29060-410. Telefax: (027) 327-9948. e-mail: angiolab@jbs.com.br
} 
Barros F S, Pontes S M, LimaM L, Henrique J S, Roldi M L, Reis F, Carone Jr. J, Moisés S-Mapeamento da safena interna com ecocolor Doppler no pré-operatório de cirurgia de revascularização miocárdica. $\quad$ Rev Bras Cir Cardiovasc 1999;14 (4): 303-7.

cientes selecionados para cirurgia de revascularização. Esse mapeamento é capaz de informar com segurança a existência ou não da safena interna, se patente, a medida do diâmetro interno em diferentes segmentos, a identificação de ramos e perfurantes nos casos de revascularização periférica e, ainda, avaliar se o sistema venoso profundo está patente. O objetivo do nosso estudo foi avaliar o papel do ecocolor Doppler e a sua utilidade no mapeamento da safena interna no pré-operatório de cirurgia de revascularização miocárdica, em uma análise conjunta com a equipe de cirurgia cardíaca.

\section{CASUÍSTICA E MÉTODOS}

Foram estudados, no período de março de 1995 a maio de 1998, 208 extremidades inferiores de 104 pacientes selecionados para a cirurgia de revascularização miocárdica (62 eram homens e 42 mulheres com idades variando entre 39 e 81 anos).

Todos os pacientes foram submetidos ao ecocolor Doppler de membros inferiores para o mapeamento da safena interna.

Os pacientes foram examinados em decúbito dorsal, na posição de Trendelenburg reverso, para analisar se o sistema venoso profundo e a safena interna estão pérvios.

Os diâmetros internos da safena foram medidos na junção com a veia femoral, no terço superior, médio e inferior da coxa e perna, utilizando-se cortes transversais pelo modo $\mathrm{B}$ (bidimensional) com o paciente em ortostase. O critério de pérvio tanto do sistema venoso profundo como da safena interna foi baseado na compressibilidade do vaso e presença de fluxo ao mapeamento colorido e Doppler pulsado. O garrote foi utilizado somente quando a veia tinha o diâmetro inferior a $3,0 \mathrm{~mm}$. O aparelho utilizado foi o ATL - HDI 3000 (Advanced Technology Laboratories) e o Acuson - Sequóia 512 com transdutores lineares de 7,5 Mhz. O trajeto da safena interna na coxa e perna foi representado de forma esquemática com os diâmetros sendo anotados paralelamente ao vaso (Figura 1). Os critérios utilizados para seleção da safena interna como apropriada para ponte aortocoronária foram o diâmetro interno maior ou igual a 3,0 mm e quando patente $(3,4)$ (Figuras 2 e 3). A safena interna foi considerada não apropriada (Figura 4) quando apresentava diâmetro interno inferior a 3,0 mm, alterações parietais, como trombose antiga, ou quando o sistema venoso profundo estava ocluído devido à presença de processo trombótico. Os parâmetros considerados pela equipe de operação cardíaca foram: a correlação anatômica do diâmetro da safena interna avaliada pelo ecocolor Doppler com a encontrada durante a exploração cirúrgica; o tempo de operação, compa-



Fig. 1 - Demonstração esquemática do mapeamento da safena interna

rado àquele em que a procura aleatória da safena se faz necessária, a maior segurança da equipe em relação à certeza da qualidade do vaso e a satisfação do paciente em relação à estética no pósoperatório, uma vez que a retirada da veia pode ser feita de forma escalonada com menor trauma, redução do tamanho da incisão e, na grande maioria dos casos, com abordagem de apenas um membro.

\section{RESULTADOS}

Dentre as 208 extremidades estudadas, 186 $(89,4 \%)$ safenas foram consideradas apropriadas

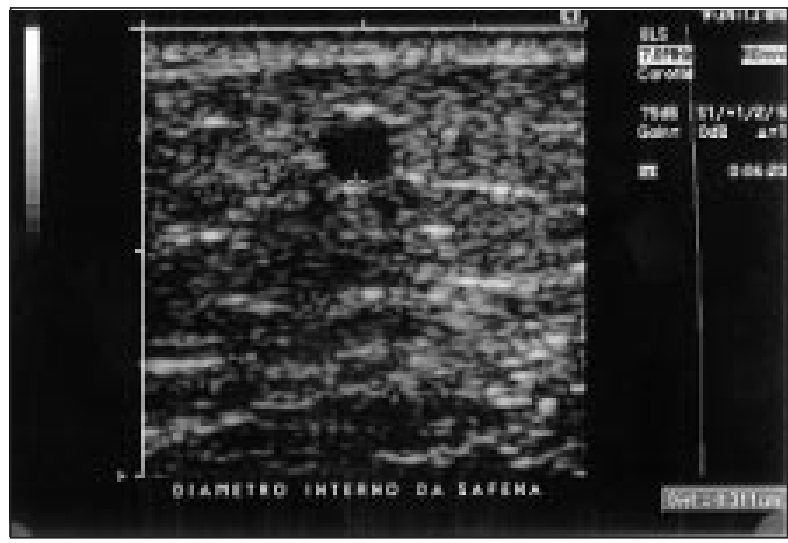

Fig. 2 - Medida do diâmetro interno da safena pelo modo B. 
Barros F S, Pontes S M, LimaM L, Henrique J S, Roldi M L, Reis F, Carone Jr. J, Moisés S-Mapeamento da safena interna com ecocolor Doppler no pré-operatório de cirurgia de revascularização miocárdica. $\quad$ Rev Bras Cir Cardiovasc 1999;14 (4): 303-7.

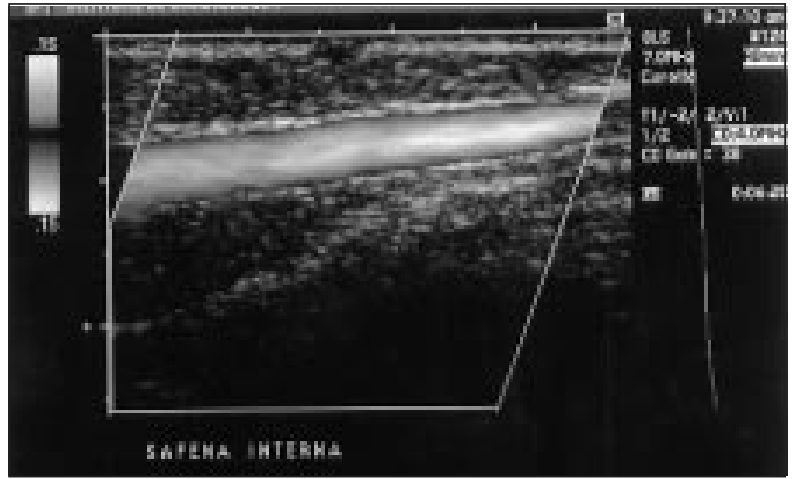

Fig. 3 - Avaliação da presença de fluxo da safena interna pelo mapeamento colorido.

para utilização como ponte aortocoronária. Em 17 $(8,2 \%)$ extremidades, a safena interna não preenchia os critérios de seleção, sendo $12(70,5 \%)$ pela presença de diâmetro inferior a 2,0 mm, 4 (23,5\%) pela presença de dilatação varicosa e comprometimento parietal difuso e $1(6,0 \%)$ em que o sistema venoso profundo estava ocluído por trombose antiga. Em 5 (24\%) extremidades o exame foi compatível com safenectomia radical. Estes últimos pacien-
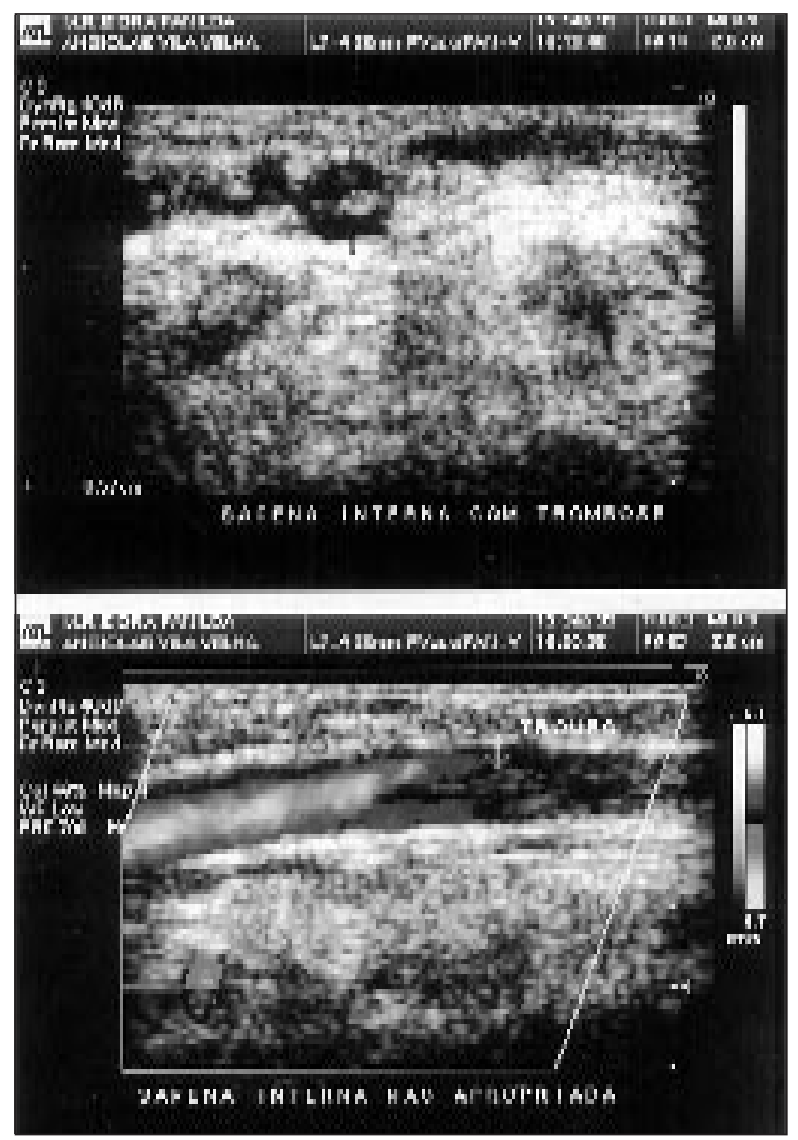

Fig. 4 - Safena interna não apropriada pela presença de trombo mural. tes foram encaminhados para a realização do exame, pois desconheciam o tipo de operação realizada anteriormente para o tratamento de varizes.

A avaliação da equipe cirúrgica durante a exploração da safena foi concordante com o estudo pelo ecocolor Doppler: vaso patente e qualidade do vaso. A análise do diâmetro foi um pouco dificultada, pois o ecografista mede o diâmetro interno do vaso e, durante a exploração cirúrgica, é medido o diâmetro externo após distensão com solução salina. Essa observação sugere uma subestimação da medida do diâmetro do vaso pelo ultra-som. Entretanto, em nenhum caso em que a safena interna foi selecionada pelo ecocolor Doppler como apropriada, deixou de ser utilizada como ponte aortocoronária. Em contrapartida, em 2 pacientes em que o ecocolor Doppler classificou a safena interna como não apropriada (uma safena por evidenciar diâmetro inferior a 2,0 mm e outra por apresentar dilatação maior do que $5,0 \mathrm{~mm}$ e alterações parietais segmentares), a exploração cirúrgica confirmou o achado ultrasonográfico. No primeiro caso, o cirurgião descreveu a veia como muito fina, não sendo possível a sua utilização como ponte. No segundo, a veia foi utilizada, sendo que, no primeiro dia de pós-operatório, a paciente apresentou sinais compatíveis com infarto agudo do miocárdico e a angiocoronariografia evidenciou oclusão da ponte.

\section{COMENTÁRIOS}

A safena interna é considerada, tanto pelo cirurgião cardíaco como pelo cirurgião vascular, uma boa opção para utilização como conduto vascular nas operações de revascularização. Para isso, o conhecimento da anatomia, se patente, o diâmetro e o comprimento da veia são fatores cruciais para determinar o sucesso de uma ponte. O diâmetro mínimo considerado ideal é de $3,0 \mathrm{~mm}$, segundo PRIEST \& ZWIEBEL (3) e $2,5 \mathrm{~mm}$ de acordo com POLAK ${ }^{(4)}$. O ecocolor Doppler permite, sem riscos para o paciente ou dano no segmento venoso, informar ao cirurgião o estado anatômico e funcional das veias passíveis de serem selecionadas para a ponte. Alguns autores, em estudo comparativo entre o mapeamento venoso pelo ecocolor Doppler e a avaliação per-operatória, relatam uma subestimação no diâmetro medido pelo ultra-som (5). HEAD \& BROWN (6) compararam o diâmetro interno medido pelo ecocolor Doppler de 100 safenas internas e externas com o diâmetro externo da veia distendida no per-operatório da operação de revascularização miocárdica e relataram que a medida per-operatória é aproximadamente 1,5 $\mathrm{mm}$ maior do que a avaliada pelo ecocolor Doppler. SALLES-CUNHA et al. (7) relataram uma diferença de aproximadamente 2,0 $\mathrm{mm}$ a mais na medida do diâmetro externo das 
Barros F S, Pontes S M, LimaM L, Henrique J S, Roldi M L, Reis F, Carone Jr. J, Moisés S-Mapeamento da safena interna com ecocolor Doppler no pré-operatório de cirurgia de revascularização miocárdica. Rev Bras Cir Cardiovasc 1999;14 (4): 303-7.

veias cefálica e basílica durante a operação, em relação ao mapeamento prévio com ultra-som. Por sua vez, KAREN et al. (8), estudando a utilidade do mapeamento venoso com ecocolor Doppler no préoperatório de operações de revascularização miocárdica, periférica e realização de fístula arteriovenosa para hemodiálise, compararam o diâmetro, a profundidade, a identificação de ramos e a marcação do trajeto anatômico do vaso na pele encontrados no estudo ecográfico com a impressão dos cirurgiões em 79 veias exploradas cirurgicamente. Setenta (95\%) das veias exploradas foram consideradas apropriadas, havendo uma acurácia de 95\% a 97\% em relação aos parâmetros analisados. A marcação de ramos e a avaliação da profundidade do vaso foram consideradas de pouca utilidade. $\mathrm{Na}$ nossa casuística, os achados foram semelhantes aos encontrados na literatura, tendo havido por parte da equipe cirúrgica uma observação a respeito da subestimação do diâmetro pelo ecocolor Doppler de aproximadamente 1 a $1,5 \mathrm{~mm}$. A identificação de ramos e perfurantes necessária ao mapeamento venoso antes da operação de revascularização periférica não foi analisada neste estudo por ser uma informação desnecessária. Como descrito na literatura, os casos em que o ecocolor Doppler classifica a safena interna como não apropriada para ser utilizada como conduto vascular, a persistência de usá-la com este objetivo é deletéria para o paciente, visto que em um dos pacientes desta casuística a utilização da safena interna classificada pelo ecocolor Doppler como não apropriada, foi considerada como o motivo principal de oclusão da ponte aortocoronária no pós-operatório.

Assim, a questão mais importante a ser analisada em relação aos estudos referentes a este tema é se o vaso é considerado apropriado ou não para ser utilizado como substituto arterial. A avaliação com ecocolor Doppler comparada com a impressão do cirurgião confirma a eficácia do método.

\section{CONCLUSÃO}

Os autores concluem que o ecocolor Doppler, sendo um método diagnóstico não invasivo, pode ser útil no mapeamento da safena interna no préoperatório da operação de revascularização miocárdica, devido à sua capacidade de demonstrar com segurança a presença e o trajeto da safena interna, se patente e a medida do seu diâmetro em diversos pontos, permitindo ao cirurgião cardíaco um planejamento cirúrgico em que a equipe já tenha o conhecimento prévio do membro a ser abordado durante 0 ato cirúrgico, a certeza da qualidade do vaso a ser retirado, a preservação estética e, finalmente, a possibilidade da preparação pré-operatória de outra opção, naqueles pacientes em que a safena interna não for considerada apropriada.

RBCCV 44205-472

Barros F S, Pontes S M, Lima M L, Henrique J S, Roldi M L, Reis F, Carone Jr. J, Moisés S. - Echocolor Doppler saphenous vein mapping prior to myocardial revascularization. Rev Bras Cir Cardiovasc1 999;14 (4): 303-7.

ABSTRACT: Introduction: The vascular echocolor Doppler, has made possible the observation of the greater saphenous vein (GSV) state without additional risk to the patient or to vascular lesions. The objective of the study was, working with a group of cardiovascular surgeons, to show the utility of the mapping of the GSV before coronary artery bypass graft (CABG) surgery.

Method: During the period from March 1995 to May 1998, 208 extremities of 104 patients selected for CABG were studied. The criteria used for the selection of the GSV was the patency and a diameter larger than or equal to $3.0 \mathrm{~mm}$, using the the ultra-sound equipment ATL - HDI 3000 and Acuson-Sequóia 512.

Results: Within the 208 extremities studied, 186 (89.4\%) were appropriate for CABG in accordance with the description from the echocolor Doppler, seventeen (8.2\%) did not fulfill the criteria, and five (2.4\%) showed GSV stripping. Two of the seventeen inappropriate GSV were surgically explored, confirming the echoultrasound findings.

Conclusions: The authors conclude that the echocolor Doppler can be effective in the mapping of GSV in the preoperative examination of the greater saphenous vein and its selection for CABG. Due to it's capacity to correctly identify and select veins for by - pass surgery, the echocolor Doppler saves time in the search for veins and permits the surgeon to better plan the surgery.

DESCRIPTORS: Myocardial revascularization. Echocardiography, Doppler color. Saphenous vein, ultrasonography. Preoperative care, methods. 


\section{REFERÊNCIAS BIBLIOGRÁFICAS}

1 Veith F J, Moss C M, Sprayregen S, Montefusco C Preoperative saphenous venography in arterial reconstructive surgery of the lower extremity. Surgery 1979; 79: 253-6.

2 Leopold P W, Shandall A A, Corson J D, Shah D M, Leather R P, Karmody A M - Inicial experience comparing B-mode imaging and venography of the saphenous vein before in situ bypass. Am J Surg 1986; 152: 206-10.

3 Priest D L \& Zwiebel W J - Insuficiência venosa crônica, veias varicosas e mapeamento da veia safena. In: Zwiebel WJ ed. Introdução à ultrasonografia vascular. 3. ed. Rio de Janeiro: Revinter, 1996: 319-27.
$4 \quad$ Polak J F - Peripheral vascular sonography: a practical guide. Baltimore, W. Wilkins, 1992: 321-3.

5 Chang B B, Paty P S, Shah D M, Leather P R - The lesser saphenous vein: an underappreciated source of autogenous vein. J Vasc Surg 1992; 15: 152-7.

6 Head H D \& Browm M E - Preoperative vein mapping for coronary artery bypass operations. Ann Thorac Surg 1995; 59:144-8.

7 Salles-Cunha S X, Andros G, Harris R W, Dulawa L B, Oblat R W - Preoperative noninvasive assessment of arms veins to be used as bypass grafts in the lower extremities. J Vasc Surg 1986; 3: 813-6.

8 Cousens K A, Altemus A P, Musson A M, Zwolak R M - Utility of preoperative vein mapping. J Vasc Technol 1997; 21: 227-31 